\title{
Estudo das alterações do ponto B durante o tratamento ortodôntico*
}

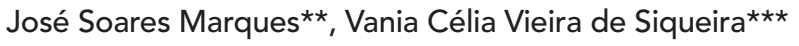

\section{Resumo}

Objetivo: os autores desse trabalho estudaram e compararam as possiveis alterações na região do ponto B, decorrentes do tratamento ortodôntico, em casos tratados com e sem extração dos quatro primeiros pré-molares. Metodologia: foram avaliadas, cefalometricamente, trinta jovens do gênero feminino, leucodermas, dolicofaciais, com má oclusão do tipo Classe II, primeira divisão, e idade média de 12,3 anos ao início do tratamento. Quinze jovens foram submetidas à extração dos quatro primeiros pré-molares e as outras 15 não. Foram mensuradas as grandezas cefalométricas relativas ao IMPA, 1-ND, B-ND e Bperp-GoMe e os dados obtidos foram submetidos à análise de Kruskall-Wallis e aos testes de Friedman e de correlação de Pearson. Resultados: os resultados para a medida IMPA mostraram que ocorreu uma tendência de aumento desta medida no grupo sem extrações e uma expressiva diminuição no grupo com extrações. A medida 1-ND apresentou uma diminuição no grupo com extrações e um suave aumento no grupo sem extrações. Ocorreu uma tendência à diminuição da medida B-ND no grupo com extrações e de inalteração no grupo sem extrações, mas não ocorreram diferenças estatisticamente significantes intergrupos. A medida Bperp-GoMe, em ambos os grupos, aumentou na avaliação do pós-tratamento. Ocorreu uma correlação positiva entre os valores do IMPA, 1-ND e B-ND no grupo com extrações, pois uma diminuição do IMPA implicou em diminuição do 1-ND e em uma localização mais para posterior do ponto B. Conclusões: esses resultados demonstraram que as alterações do ponto B, no sentido ântero-posterior, decorrentes da movimentação ortodôntica, indicaram uma tendência para a lingualização nos pacientes submetidos a extrações. No sentido vertical, as alterações no ponto B, em ambos os grupos, mostraram significância, uma vez que a medida no exame final encontrava-se significativamente superior à medida do exame inicial.

Palavras-chave: Ponto B. Tratamento ortodôntico. Extrações. Classe II. Remodelação mandibular.

\footnotetext{
* Resumo da dissertação de mestrado apresentada ao curso de pós-graduação em Ortodontia da Pontifícia Universidade Católica de Minas Gerais PUC/Minas.

** Mestre em Ortodontia pela Pontifícia Universidade Católica de Minas Gerais PUC/Minas.

*** Professora Assistente Doutora da disciplina de Ortodontia da Faculdade de Odontologia de Piracicaba UNICAMP. Professora Adjunta III da disciplina de Ortodontia da Pontifícia Universidade Católica de Minas Gerais PUC/Minas.
} 


\section{INTRODUÇÃO E REVISÃO DA LITERATURA}

Inúmeros pesquisadores empenharam-se em obter informações sobre os processos e os mecanismos envolvidos no crescimento e no desenvolvimento craniofacial, de forma a guiá-los durante o diagnóstico, planejamento do tratamento ortodôntico e estabilidade do mesmo $5,6,7,10,18,24,25,26$.

As dificuldades na condução das pesquisas esbarraram na constatação de que o ser vivo mantém-se em constantes mudanças, sendo suas estruturas ósseas instáveis ${ }^{3,8,13,19,28}$. Além disso, a variabilidade individual dificulta a previsibilidade, pois um mesmo estímulo provoca respostas diferentes em organismos distintos ${ }^{14,15,20}$.

As alterações decorrentes do crescimento e do desenvolvimento craniofacial não cessam totalmente após a adolescência e valores convencionalmente adotados como representativos de normalidade nem sempre podem ser empregados indistintamente durante o diagnóstico e planejamento dos tratamentos ${ }^{21,27}$.

Apesar da importância clínica, existem poucas informações pertinentes sobre as alterações de crescimento na região da sínfise mandibular, com objetivo de fornecer dados que possam ser usados no planejamento e na avaliação do tratamento ortodôntico. Estudos longitudinais dessa região, realizados em crianças e adolescentes não tratados ortodonticamente, observaram que as mudanças de crescimento no plano vertical ocorreram principalmente nos pontos localizados na região mais alta da crista alveolar da sínfise e a taxa de crescimento vertical encontrava-se maior durante a puberdade. A velocidade de crescimento vertical para o ponto $\mathrm{B}$ direcionou-se superiormente, mostrando uma maior alteração horizontal para o gênero masculino, movendo lingualmente mais de $2 \mathrm{~mm}$ durante o período de observação de 8 anos. No gênero feminino as maiores taxas de movimento lingual ocorreram para os pontos ântero-superiores da sínfise. Durante a puberdade, os incisivos inferiores, no gênero feminino, moveram-se lingualmente, mas no gênero masculino os incisivos mantiveram sua posição $0^{7,9,19}$.
Durante o processo de crescimento ocorrem alterações na região da sínfise mentoniana e, conseqüentemente, modificações na posição do incisivo inferior ${ }^{17}$. Avaliando-se telerradiografias seriadas, obtidas em norma lateral, de 22 gêmeos idênticos, de ambos os gêneros, nas idades de 6, 8, 10 e 15 anos, observa-se que, na idade entre 6 e 7 anos, a sínfise mentoniana inicia um surto de crescimento e aumenta em sua dimensão ântero-posterior, com os jovens do gênero masculino apresentando um aumento maior do que os do feminino. $\mathrm{Na}$ idade entre 8 e 9 anos, a taxa de crescimento da sínfise diminui, voltando a apresentar um novo surto dos 10 aos 12 anos. As alterações no ponto B ocorrem suavemente dos 6 aos 10 anos e de forma mais marcada dos 10 aos 15 anos, concomitantemente com a inclinação lingual do incisivo inferior. $\mathrm{O}$ dimorfismo sexual relacionado ao ponto B ocorre de forma quantitativa e não qualitativa, sendo que os jovens do gênero masculino apresentam 2 vezes mais alterações na espessura da sínfise mandibular dos 10 aos 15 anos $^{17}$.

Existe uma forte correlação entre a morfologia da sínfise e a direção de crescimento mandibular no gênero masculino, quando comparado ao feminino. A proporção entre a profundidade e a altura da sínfise encontra-se fortemente relacionada com a direção de crescimento mandibular no gênero masculino. Em ambos os gêneros, a sínfise mandibular com direção de crescimento anterior possui pouca altura, grande profundidade e pequena proporção entre altura e profundidade. Por outro lado, uma sínfise com grande altura, pouca profundidade e grande proporção entre altura e profundidade aponta para uma direção de crescimento mandibular posterior. Ocorre uma contínua mudança na sínfise mandibular na fase adulta em ambos os gêneros, porém em intensidade menor e numa época mais precoce no gênero feminino, se comparado com o masculino ${ }^{1}$.

$\mathrm{O}$ aumento em altura da sínfise mandibular parece coincidir com um estreitamento na forma, entretanto, não alterando significativamente a área 
total da sínfise. Em pacientes com face longa, as dimensões sagitais na região apical dos incisivos inferiores encontram-se reduzidas, em contraste com pacientes de face curta, nos quais estas dimensões mostram-se aumentadas. $\mathrm{O}$ estudo detalhado da morfologia da sínfise mandibular auxilia na predição da altura facial ântero-inferior na fase adulta ${ }^{4}$.

Existe uma significante, mas complexa, relação entre as estruturas do corpo mandibular e o tipo facial. A espessura da cortical óssea vestibular e lingual, avaliada em crânios secos de asiáticos do gênero masculino, encontrava-se maior no grupo com faces curtas do que no grupo com faces médias e longas. A espessura da cortical óssea basal, na região dos incisivos inferiores apresentava-se maior no grupo de faces curtas do que no de faces longas, encontrando-se igual nos três grupos na região dos pré-molares ${ }^{35}$.

Com o intuito de quantificar as alterações provocadas pela remodelação óssea na região do côndilo, gônio e sínfise mandibular, representada pelos pontos mentoniano, pogônio e ponto B, Baumrind et al. ${ }^{2}$, em 1992, avaliaram uma amostra de 31 telerradiografias, em norma lateral, de jovens que receberam implantes metálicos e foram acompanhados anualmente dos 8,5 anos aos 15,5 anos de idade. Desses, 18 receberam tratamento ortodôntico e 13 não. Os autores observaram que o pogônio e o mentoniano se deslocaram levemente para baixo e para trás, sendo que as alterações no mentoniano ocorreram pela aposição óssea na superfície inferior da sínfise. As alterações ocorridas no ponto $\mathrm{B}$ encontravam-se maiores do que aquelas observadas para o pogônio e o mentoniano. Ocorreu uma reabsorção óssea na região do ponto $\mathrm{B}$, que se deslocou para cima e para trás, causada pelo desenvolvimento vertical do processo alveolar e pela erupção dos dentes ântero-inferiores. Os autores confirmaram a evidência de reabsorção óssea na superfície anterior da mandíbula.

Os processos alveolares modificam-se de maneira marcante, à medida que os dentes posicionam-se ortodonticamente, e continuam a se ajustar durante e após o período de contenção. As alterações na posição dos incisivos inferiores, produzidas pelo tratamento ortodôntico, afetam a posição do ponto $\mathrm{B}$, tornando-se necessário $\mathrm{O}$ estabelecimento de um ponto mais estável para a estimativa do posicionamento ântero-posterior mandibular, como o ponto $\mathrm{D}^{30}$.

Nos casos onde a sínfise mandibular apresentase estreita e alta, os movimentos sagitais pronunciados e de rotação de incisivos, provocados pelos aparelhos ortodônticos fixos, conduzem a uma perda óssea progressiva da cortical lingual e vestibular, causando iatrogenias graves ${ }^{38}$.

Após compulsada a literatura pertinente ao assunto, os autores propuseram estudar a remodelação óssea na região do ponto $\mathrm{B}$, decorrente da movimentação dos incisivos inferiores, em jovens, inicialmente com má oclusão do tipo Classe II, $1^{\text {a }}$ divisão, leucodermas, dolicofaciais, submetidos ao tratamento ortodôntico com extração dos quatros primeiros pré-molares e uso do aparelho extrabucal combinado (grupo experimental). Compararam os dados cefalométricos obtidos aos de um grupo de jovens com as mesmas características, mas que submeteram-se ao tratamento ortodôntico corretivo sem extrações (grupo controle).

\section{MATERIAL E MÉTODOS Material}

Esse trabalho científico respeitou todos os requisitos exigidos pelo Comitê de Ética em Pesquisa da Pontifícia Universidade Católica de Minas Gerais para a sua realização, obtendo a aprovação.

Do acervo de documentação ortodôntica pertencente à disciplina de Ortodontia da Faculdade de Odontologia da PUC/Minas, analisou-se as telerradiografias, realizadas em norma lateral, de 748 jovens que se submeteram ao tratamento ortodôntico corretivo, na referida disciplina.

Elegeu-se 30 jovens, leucodermas, da região de Belo Horizonte/MG, do gênero feminino, que apresentavam, inicialmente, má oclusão do tipo Classe II, $1^{\text {a }}$ divisão, e que possuiam o ângulo 
SN.GoGn maior que $32^{\circ}$, identificando-se assim o tipo facial vertical, dolicofacial, segundo os preceitos de Steiner ${ }^{29,30}$. O tratamento ortodôntico realizou-se empregando-se a técnica do arco de canto, associado ao aparelho extrabucal combinado com tração alta, sendo que 15 jovens trataram-se com extração dos quatro primeiros pré-molares, caracterizando o grupo experimental, e 15 jovens sem extrações, caracterizando o grupo controle.

Efetuou-se as radiografias no início e no término do tratamento, totalizando 60 telerradiografias.

\section{Métodos}

Das pacientes que submeteram-se à extração dos quatro primeiros pré-molares durante o tratamento ortodôntico (grupo experimental) foram selecionadas apenas as telerradiografias iniciais que apresentassem os mesmos e as telerradiografias finais com ausência desses dentes. Das pacientes que não realizaram extrações (grupo controle) foram selecionadas as telerradiografias iniciais e finais em que estes dentes estavam presentes.

Durante a elaboração dos cefalogramas, apoiados nos postulados de Downs ${ }^{11,12}$, Riedel ${ }^{22,23}$, Steiner $^{29,30}$, Tweed ${ }^{31,32,33,34}$ e Wylie ${ }^{37}$, identificou-se as estruturas dentoesqueléticas e do perfil tegumentar, bem como a demarcação dos pontos cefa- lométricos delineando-se os planos e linhas que auxiliaram na avaliação das grandezas: B-ND, 1-ND, Bperp-GoMe, SN.GoGn e IMPA (Fig. 1, 2, 3).

\section{Erro do método}

De forma a aumentar o grau de confiabilidade e exatidão traçou-se cada telerradiografia duas vezes, pelo mesmo pesquisador devidamente e previamente calibrado, em duas épocas distintas, com 45 dias entre cada traçado, obtendo-se dois valores para cada variável cefalométrica. Por meio do cálculo de média aritmética simples, obtevese um valor médio de cada medida, sendo então empregado para as análises estatísticas. Elegeu-se e novamente traçou-se, aleatoriamente, 10 telerradiografias e os valores cefalométricos obtidos foram submetidos à fórmula de Dahlgerg ${ }^{16,36}$ avaliando-se a existência ou não de erros do método.

\section{ANÁLISE ESTATÍSTICA}

Após a obtenção dos dados de interesse ao estudo, empregou-se as seguintes análises estatísticas:

1) Análises descritivas, obtendo-se valores máximos, médios, mínimos e desvio-padrão. $\mathrm{O}$ valor de $\mathrm{n}$ refere-se ao tamanho da amostra avaliada.

2) Teste de Kruskal-Wallis, para realizar algumas comparações entre os grupos com e sem extrações.

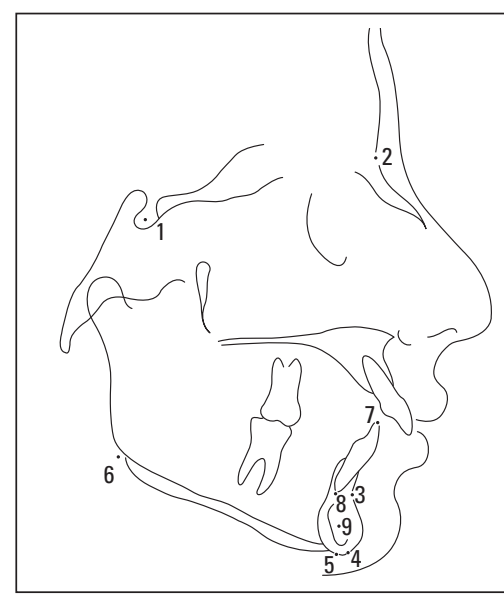

FIGURA 1 - Identificação das estruturas dentoesqueléticas, do perfil tegumentar e dos pontos cefalométricos utilizados.

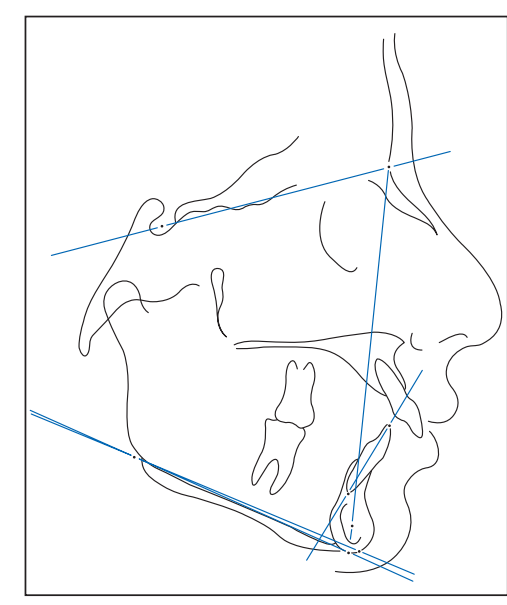

FIGURA 2 - Demarcação dos planos e linhas de referência.

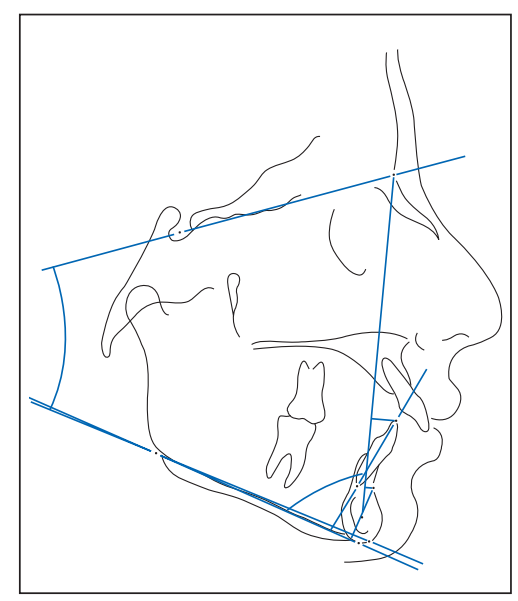

FIGURA 3 - Identificação das grandezas cefalométricas lineares e angulares. 
3) Teste de Friedman, para a comparação entre os dois tempos (antes e depois) com relação às medidas das variáveis de interesse.

4) Análise de correlação de Pearson (r), para observar a relação entre as medidas das variáveis.

Todos os resultados foram considerados signi-

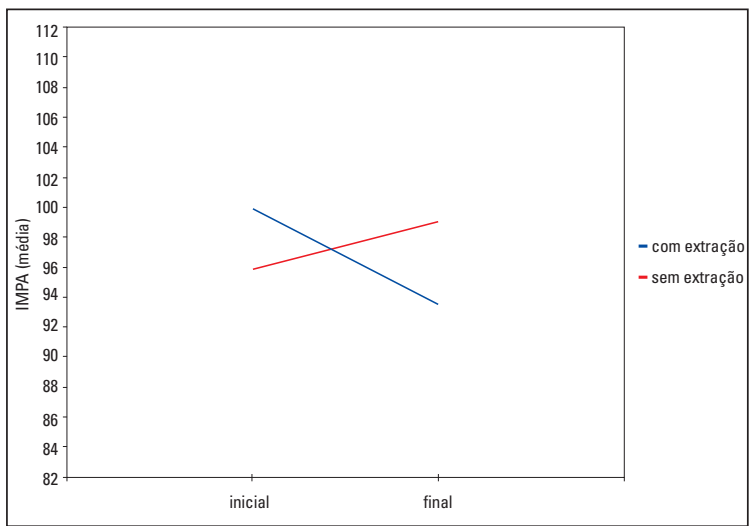

GRÁFICO 1 - Avaliação do IMPA ao início e final do tratamento ortodôntico, nos grupos com e sem extrações.

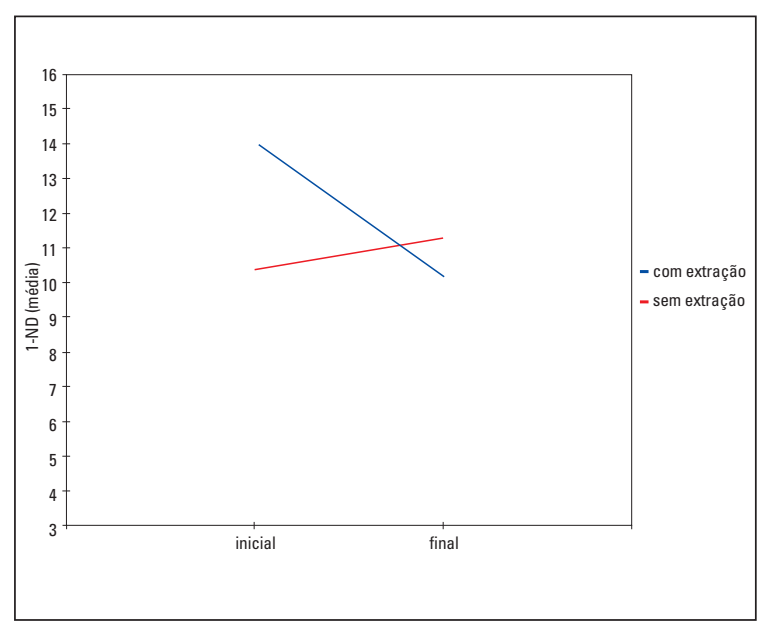

GRÁFICO 3 - Avaliação do 1-ND ao início e final do tratamento ortodôntico, nos grupos com e sem extrações.

\section{DISCUSSÃO}

Avaliando-se as alterações do ângulo IMPA (Gráf. 1, Tab. 1), observa-se que ocorreu uma diminuição de seus valores no grupo que submeteuse às extrações e um aumento de seus valores no ficativos para uma probabilidade de significância inferior a $5 \%(\mathrm{p}<0,05)$.

\section{RESULTADOS}

Os resultados encontram-se nas tabelas 1 a $8 \mathrm{e}$ gráficos 1 a 4 .

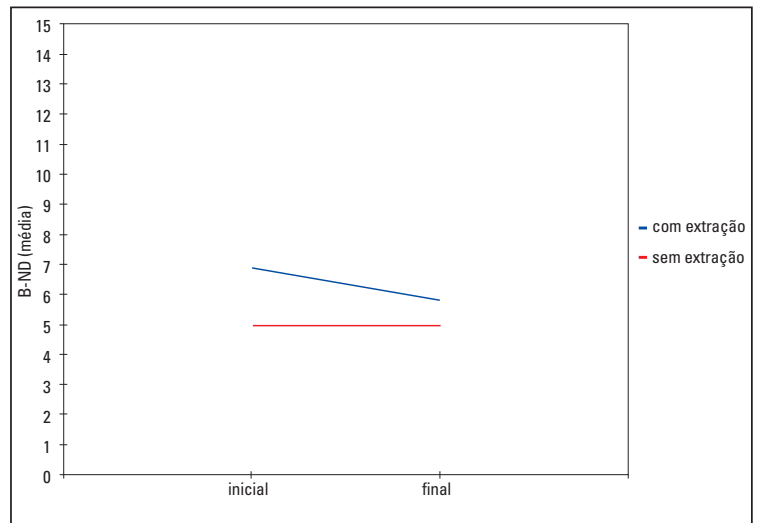

GRÁFICO 2 - Avaliação do B-ND ao início e final do tratamento ortodôntico, nos grupos com e sem extrações.

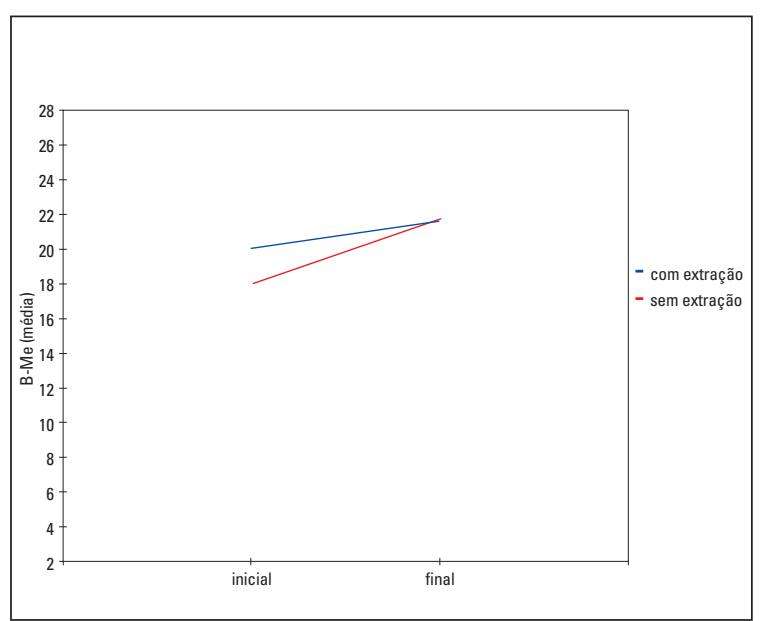

GRÁFICO 4 - Avaliação do Bperp-GoMe ao início e final do tratamento ortodôntico, nos grupos com e sem extrações.

grupo tratado sem extrações. Informações semelhantes às obtidas por outros pesquisadores em estudos realizados anteriormente ${ }^{5,25,26,27}$.

Ocorreu uma diminuição dos valores relativos à medida B-ND após o tratamento no grupo com 
Tabela 1 - Análise comparativa da medida IMPA quanto ao tempo e ao grupo de procedência.

\begin{tabular}{|c|c|c|c|c|c|c|c|c|c|c|c|c|}
\hline \multirow{3}{*}{ grupo } & \multicolumn{5}{|c|}{ inicial } & \multicolumn{5}{|c|}{ final } & \multirow[b]{3}{*}{$\mathbf{p}$} & \multirow{3}{*}{ conclusão } \\
\hline & \multicolumn{5}{|c|}{ medidas descritivas } & \multicolumn{5}{|c|}{ medidas descritivas } & & \\
\hline & mín. & máx. & mediana & média & d.p. & mín. & máx. & mediana & média & d.p. & & \\
\hline com extrações & 84,0 & 112,0 & 98,0 & 99,9 & 7,7 & 83,0 & 102,0 & 95,0 & 93,5 & 5,1 & 0,001 & $\mathrm{I}>\mathrm{F}$ \\
\hline sem extrações & 82,0 & 108,0 & 96,0 & 95,9 & 7,9 & 85,0 & 110,0 & 99,0 & 99,0 & 7,1 & 0,052 & $\mathrm{I}=\mathrm{F}$ \\
\hline $\mathbf{p}$ & & & 0,104 & & & & & 0,023 & & & & \\
\hline conclusão & & & $C=S$ & & & & & $\mathrm{C}<\mathrm{S}$ & & & & \\
\hline
\end{tabular}

Nota: 0 valor $p$ horizontal refere-se ao teste Kruskal-Wallis, o valor $p$ vertical refere-se ao teste Friedman.

C - com extração, S - sem extração, I - inicial, F - final.

Tabela 2 - Análise comparativa da medida B-ND quanto ao tempo e ao grupo de procedência.

\begin{tabular}{|c|c|c|c|c|c|c|c|c|c|c|c|c|}
\hline \multirow{3}{*}{ grupo } & \multicolumn{5}{|c|}{ inicial } & \multicolumn{5}{|c|}{ final } & \multirow[b]{3}{*}{$\mathbf{p}$} & \multirow{3}{*}{ conclusão } \\
\hline & \multicolumn{4}{|c|}{ medidas descritivas } & & \multicolumn{5}{|c|}{ medidas descritivas } & & \\
\hline & mín. & máx. & mediana & média & d.p. & mín. & máx. & mediana & média & d.p. & & \\
\hline com extrações & 4,0 & 14,5 & 7,0 & 6,9 & 2,5 & 2,0 & 8,0 & 6,0 & 5,8 & 1,7 & 0,083 & $\mathrm{I}=\mathrm{F}$ \\
\hline sem extrações & 0,0 & 8,0 & 5,5 & 5,0 & 2,0 & $-2,0$ & 8,0 & 5,0 & 5,0 & 2,5 & 0,527 & $\mathrm{I}=\mathrm{F}$ \\
\hline $\mathbf{p}$ & & & 0,050 & & & & & 0,473 & & & & \\
\hline conclusão & & & $\mathrm{C}=\mathrm{S}$ & & & & & $\mathrm{C}=\mathrm{S}$ & & & & \\
\hline
\end{tabular}

Nota: 0 valor $\mathrm{p}$ horizontal refere-se ao teste Kruskal-Wallis, 0 valor $\mathrm{p}$ vertical refere-se ao teste Friedman.

C - com extração, S - sem extração, I - inicial, F - final.

Tabela 3 - Análise comparativa da medida 1-ND quanto ao tempo e ao grupo de procedência.

\begin{tabular}{|c|c|c|c|c|c|c|c|c|c|c|c|c|}
\hline \multirow{3}{*}{ grupo } & \multicolumn{5}{|c|}{ inicial } & \multicolumn{5}{|c|}{ final } & & \multirow{3}{*}{ conclusão } \\
\hline & \multicolumn{5}{|c|}{ medidas descritivas } & \multicolumn{5}{|c|}{ medidas descritivas } & \multirow[b]{2}{*}{$\mathbf{p}$} & \\
\hline & mín. & máx. & mediana & média & d.p. & mín. & máx. & mediana & média & d.p. & & \\
\hline com extrações & 9,0 & 17,0 & 14,0 & 14,0 & 2,3 & 4,0 & 16,0 & 10,0 & 10,2 & 3,0 & $<0,001$ & $\mathrm{I}>\mathrm{F}$ \\
\hline sem extrações & 3,5 & 15,0 & 12,0 & 10,4 & 4,2 & 4,0 & 16,0 & 13,0 & 11,3 & 3,6 & 0,033 & $\mathrm{I}<\mathrm{F}$ \\
\hline $\mathbf{p}$ & & & 0,006 & & & & & 0,279 & & & & \\
\hline conclusão & & & $C>S$ & & & & & $C=S$ & & & & \\
\hline
\end{tabular}

Nota: 0 valor $p$ horizontal refere-se ao teste Kruskal-Wallis, o valor $\mathrm{p}$ vertical refere-se ao teste Friedman.

C - com extração, S - sem extração, I - inicial, F - final.

Tabela 4 - Análise comparativa da medida Bperp-GoMe quanto ao tempo e ao grupo de procedência.

\begin{tabular}{|c|c|c|c|c|c|c|c|c|c|c|c|c|}
\hline \multirow{3}{*}{ grupo } & \multicolumn{5}{|c|}{ inicial } & \multicolumn{5}{|c|}{ final } & & \multirow{3}{*}{ conclusão } \\
\hline & \multicolumn{5}{|c|}{ medidas descritivas } & \multicolumn{5}{|c|}{ medidas descritivas } & & \\
\hline & mín. & máx. & mediana & média & d.p & mín. & máx. & mediana & média & d.p. & $\mathbf{p}$ & \\
\hline com extrações & 16,0 & 25,0 & 20,0 & 20,0 & 2,7 & 15,0 & 27,0 & 22,0 & 21,6 & 3,1 & 0,008 & $\mathrm{I}<\mathrm{F}$ \\
\hline sem extrações & 2,0 & 22,0 & 19,0 & 18,0 & 4,7 & 18,0 & 25,0 & 21,0 & 21,7 & 1,8 & $<0,001$ & $\mathrm{I}<\mathrm{F}$ \\
\hline $\mathbf{p}$ & & & 0,271 & & & & & 0,917 & & & & \\
\hline conclusão & & & $C=S$ & & & & & $C=S$ & & & & \\
\hline
\end{tabular}

Nota: 0 valor $p$ horizontal refere-se ao teste Kruskal-Wallis, 0 valor $p$ vertical refere-se ao teste Friedman.

C - com extração, S - sem extração, I - inicial, F - final. 
Estudo das alterações do ponto B durante o tratamento ortodôntico

Tabela 5 - Análise de correlação entre as medidas iniciais do grupo com extrações.

\begin{tabular}{|c|c|c|c|c|c|c|c|c|}
\hline \multirow{2}{*}{ Medidas } & \multicolumn{2}{|c|}{ IMPA } & \multicolumn{2}{|c|}{ B-ND } & \multicolumn{2}{|c|}{ 1-ND } & \multicolumn{2}{|c|}{ Bperp-GoMe } \\
\hline & $\mathbf{r}$ & $\mathbf{p}$ & $r$ & $\mathbf{p}$ & $r$ & $\mathbf{p}$ & $r$ & $\mathbf{p}$ \\
\hline B-ND & 0,441 & 0,100 & & & & & & \\
\hline 1-ND & 0,599 & 0,018 & 0,394 & 0,146 & & & & \\
\hline Bperp-GoMe & $-0,168$ & 0,550 & 0,320 & 0,245 & 0,092 & 0,744 & & \\
\hline
\end{tabular}

Nota: o valor de $p$ refere-se à probabilidade de significância do índice de correlação (r) de Pearson.

Tabela 6 - Análise de correlação entre as medidas iniciais do grupo sem extrações.

\begin{tabular}{|c|c|c|c|c|c|c|c|c|}
\hline \multirow{2}{*}{ Medidas } & \multicolumn{2}{|c|}{ IMPA } & \multicolumn{2}{|c|}{ B-ND } & \multicolumn{2}{|c|}{ 1-ND } & \multicolumn{2}{|c|}{ Bperp-GoMe } \\
\hline & $\mathbf{r}$ & $\mathbf{p}$ & $\mathbf{r}$ & $\mathbf{p}$ & $\mathbf{r}$ & $\mathbf{p}$ & $\mathbf{r}$ & $\mathbf{p}$ \\
\hline B-ND & 0,533 & 0,041 & & & & & & \\
\hline 1-ND & 0,689 & 0,005 & 0,581 & 0,023 & & & & \\
\hline Bperp-GoMe & 0,198 & 0,479 & $-0,254$ & 0,362 & 0,485 & 0,067 & & \\
\hline
\end{tabular}

Nota: 0 valor de $p$ refere-se à probabilidade de significância do índice de correlação $(r)$ de Pearson.

Tabela 7 - Análise de correlação entre as medidas finais do grupo com extrações.

\begin{tabular}{ccccccccc}
\hline \multirow{2}{*}{ Medidas } & \multicolumn{2}{c}{ IMPA } & \multicolumn{3}{c}{ B-ND } & \multicolumn{2}{c}{ 1-ND } & \multicolumn{1}{c}{ Bperp-GoMe } \\
\cline { 2 - 9 } & $\mathbf{r}$ & $\mathbf{p}$ & $\mathbf{r}$ & $\mathbf{p}$ & $\mathbf{r}$ & $\mathbf{p}$ & $\mathbf{r}$ & $\mathbf{p}$ \\
\hline B-ND & 0,243 & 0,382 & & & & & \\
1-ND & 0,165 & 0,557 & 0,389 & 0,151 & & & \\
Bperp-GoMe & $-0,300$ & 0,277 & 0,025 & 0,929 & 0,003 & 0,991 & \\
\hline
\end{tabular}

Nota: 0 valor de $p$ refere-se à probabilidade de significância do índice de correlação (r) de Pearson.

Tabela 8 - Análise de correlação entre as medidas finais do grupo sem extrações.

\begin{tabular}{ccccccccc}
\hline \multirow{2}{*}{ Medidas } & \multicolumn{2}{c}{ IMPA } & \multicolumn{3}{c}{ B-ND } & \multicolumn{2}{c}{ 1-ND } & \multicolumn{3}{c}{ Bperp-GoMe } \\
\cline { 2 - 9 } & $\mathbf{r}$ & $\mathbf{p}$ & $\mathbf{r}$ & $\mathbf{p}$ & $\mathbf{r}$ & $\mathbf{p}$ & $\mathbf{r}$ & $\mathbf{p}$ \\
\hline B-ND & 0,385 & 0,156 & & & & & \\
1-ND & $\mathbf{0 , 6 8 2}$ & $\mathbf{0 , 0 0 5}$ & $\mathbf{0 , 7 7 9}$ & $\mathbf{0 , 0 0 1}$ & & & \\
Bperp-GoMe & 0,171 & 0,543 & $-0,117$ & 0,678 & $-0,030$ & 0,915 & \\
\hline
\end{tabular}

Nota: 0 valor de $p$ refere-se à probabilidade de significância do índice de correlação (r) de Pearson.

extrações, ou seja, ocorreu um deslocamento no sentido horizontal, para trás, do ponto B. No grupo sem extrações não observou-se diferenças significativas entre as medidas obtidas no exame inicial e final, demonstrando uma falta de deslocamento do ponto B (Gráf. 2, Tab. 2). Deve-se considerar que os pacientes da presente pesquisa possuíam o ângulo SN.GoGn aumentado, caracterizando-os como dolicofaciais e, portanto, apresentando uma sínfise mais alongada e estreita, típica de crescimento predominantemente vertical ${ }^{15}$. Empregouse no presente estudo a medida B-ND, para avaliar o deslocamento horizontal do ponto $\mathrm{B}$, enquanto outros estudos usaram as superposições dos traçados cefalométricos ${ }^{9,17}$.

Verificou-se que ocorreram diferenças signi- 
ficativas no que se refere à medida $1-N D$, tanto no grupo com extrações quanto no grupo sem extrações (Gráf. 3, Tab. 3). No grupo com extrações, essa medida diminuiu, expressivamente, mostrando que ocorreu um movimento dentário no sentido lingual, em concordância com estudos desenvolvidos anteriormente $\mathrm{e}^{5,10,25,26,27}$ e no grupo sem extrações os valores aumentaram suavemente, dados similares aos de pesquisas anteriores ${ }^{6}$.

Observou-se, por meio da medida Bperp-Go$\mathrm{Me}$, que o ponto $\mathrm{B}$ deslocou-se para cima tanto no grupo com extrações quanto no grupo sem extrações (Gráf. 4, Tab. 4), confirmando o achado de outros estudos ${ }^{2,9,17}$ que relacionaram esse deslocamento ao processo normal de remodelação da sínfise mandibular.

Analisando-se as tabelas 5, 6, 7 e 8 observouse que ocorreu uma correlação positiva entre os valores finais do IMPA, 1-ND e B-ND no grupo com extrações. A diminuição do IMPA implicou na diminuição do 1-ND e uma relação mais para posterior do ponto $\mathrm{B}$.

Apoiando-se nos dados obtidos, percebe-se a importância da avaliação da morfologia da sínfise mandibular durante o diagnóstico e planejamento do tratamento ortodôntico. As alterações dessa região mostram-se marcantes durante o crescimento e desenvolvimento craniofacial. O tratamento ortodôntico realizado em jovens nessa fase, e que necessitam se submeter a extrações dentárias, deve respeitar as informações decorrentes do processo de remodelação para se obter uma adequada harmonia facial.

A localização do ponto B altera-se, principalmente nos casos com extrações de primeiros pré-molares, descolando-se expressivamente para trás, interagindo diretamente com a estética facial pós-tratamento ortodôntico imediato e em longo prazo.

Nos casos de faces longas, apresentando estreitamento ósseo alveolar na região do ponto B anteriormente ao tratamento ortodôntico, realizações de extrações dentárias que provoquem grandes movimentos dos incisivos inferiores devem ser cuidadosamente avaliadas, evitando-se iatrogenias.

\section{CONCLUSÕES}

Considerando-se as características da amostra utilizada, a metodologia científica empregada e após a análise criteriosa dos dados obtidos no decorrer do trabalho, concluiu-se que:

- Os valores apresentados para o IMPA mostraram uma tendência de aumento do início ao final do tratamento ortodôntico no grupo sem extrações e uma expressiva diminuição no grupo com extrações.

- Ocorreu uma tendência à diminuição da medida B-ND após o tratamento ortodôntico no grupo com extrações e inalteração no grupo sem extrações.

- A medida 1-NB mostrou diferenças significativas, indicando uma diminuição no grupo com extrações e um suave aumento no grupo sem extrações.

- A medida Bperp-GoMe aumentou em ambos os grupos, na avaliação do pós-tratamento, sendo o aumento mais expressivo no grupo sem extrações.

- Ocorreu uma correlação positiva entre os valores do IMPA, 1-ND e B-ND no grupo com extrações, pois uma diminuição do IMPA implicou em uma diminuição do 1-ND e em uma relação mais para posterior do ponto $\mathrm{B}$.

- As alterações do ponto B no sentido ânteroposterior, decorrentes de movimentação ortodôntica, indicaram uma tendência para lingualização nos casos com extrações. 


\title{
Study of the point B alterations during orthodontic treatment
}

\begin{abstract}
Aim: The authors of this study evaluated and compared if alterations in point B region could occur as a result of orthodontic treatment conducted with or without the extraction of the four first premolars. Methods: Thirty young females, dolicofacials, with Class II, division 1 maloclusion, mean age of 12,3 years in the beginning of the treatment were evaluated cephalometrically. Fifteen of the young females were submitted to extractions of the four first premolars while the remaining fifteen were not. Four cephalometric measurements (IMPA, 1-ND, B-ND and BperpGoMe) were evaluated and data was analyzed by the Kruskall-Wallis test, Friedman test and Pearson's correlation. Results: The IMPA measurement had a tendency to increase within the group without extractions, but in contrast was significantly decreased within the group with extractions. The 1-ND measurement exhibited a decrease in the group with extractions and a small increase in the other group. The B-ND measurement was slightly decreased in the group with extractions, was not altered in the other group, and significant differences were not observed between these two groups. The Bperp-GoMe measurement was increased in both groups. A positive correlation was observed between IMPA, 1-ND and B-ND in the extraction group, since a decrease of the IMPA implied in a decrease of the 1-ND and a lingual localization of the point $B$. Conclusions: Together, these results showed that the orthodontic treatment lead to alterations in point $B$, towards an anteroposterior position, and a tendency of lingual movement in the extraction group. The vertical evaluation revealed significant alterations in point $B$ in both groups since measurement at the end of the treatment was significantly higher than the one in the beginning.
\end{abstract}

Key words: Point B. Orthodontic treatment. Extractions. Class II. Mandibular remodeling.

\section{REFERÊNCIAS}

1. AKI, T.; NANDA, R.; CURIE, F.; NANDA, S. K. Assessment of symphysis morphology as a predictor of the direction of mandibular growth. Am. J. Orthod. Dentofacial Orthop., St. Louis, v. 106, no. 1, p. 60-69, July 1994.

2. BAUMRIND, S. et al. Mandibular remodeling measured on cephalograms. 1 - Osseous changes relative to superimpositions on metallic implants. Am. J. Orthod. Dentofacial Orthop., St. Louis, v. 102, no. 2, p. 134-142, Aug. 1992.

3. BAUMRIND, S. et al. Lower molar and incisor displacement associated with mandibular remodeling. Angle Orthod. Appleton, v. 67, no. 2, p. 93-103, July 1997.

4. BECKMANN, S. H. et al. Alveolar and skeletal dimensions associated with lower face height. Am. J. Orthod. Dentofacial Orthop., St. Louis, v. 113, no. 5, p. 498-506, May 1998.

5. BISHARA, E. S. et al. Effects of orthodontic treatment on the growth of individuals with Class II division 1 malocclusion. Angle Orthod., Appleton, v. 64, no. 3, p. 221-230, 1994.

6. BJÖRK, A. Facial growth in man, studied with the aid of metallic implants. Acta Odontol. Scand., Oslo, v. 13, no. 9-34, 1955

7. BJÖRK, A. Variations in the growth pattern of the human mandible: longitudinal radiographic study by the implant method. J. Dent. Res., Alexandria, v. 42, no. 1, p. 400-411, 1963. Supplement.

8. BJÖRK, A.; SKIELLER, V. Facial development and tooth eruption. Am J Orthod., St. Louis, v. 62, no. 4, p. 339-383, Oct. 1972.

9. BUSCHANG, P. H. et al. Childhood and pubertal growth changes of the human symphysis. Angle Orthod., Appleton, v. 62, no. 3, p. 203-209, 1992

10. COLE, H. J. Certain results of extraction in the treatment of malocclusion. Angle Orthod., Appleton, v. 3/4, no. 18, p. 103112, July/Oct. 1948

11. DOWNS, W. B. Variations in facial relationships; their significance in treatment and prognaosis. Am. J. Orthod., St. Louis, v. 34 , no. 10 , p. $812-840$, Oct. 1948

12. DOWNS, W. B. The role of cephalometrics in orthodontic case analysis and diagnosis. Am. J. Orthod., St. Louis, v. 38, no. 3 162-182, Mar. 1952
13. ENLOW, D. H.; HARRIS, D. B. A study of the postnatal growth of the human mandible. Am. J. Orthod., St. Louis, v. 50, no. 1, p. 25-50, Jan. 1974.

14. FIELDS, H. W.; PROFFIT, W. R.; NIXON, W. L.; PHILLIPS, C. E. D.; STANEK, B. S. Facial pattern differences in long-faced children and adults. Am. J. Orthod., St. Louis, v. 85, no. 3, p. 217-223, Mar. 1984.

15. HASKELL, B. S. The human chin and relationship to mandibular morphology. Angle Orthod., Appleton, v. 49, no. 3, p. 153166, July 1979

16. HOUSTON, W. J. B. Analysis of erros in orthodontic measurements. Am. J. Orthod., St. Louis, v. 83, no. 5, p. 382-390, May 1983.

17. JONES, J. D. Accompanying development of the symphysis and point B. Angle Orthod., Appleton, v. 36, no. 4, p. 358362, Oct. 1966

18. KARSEN, A. T. Association between facial height development and mandibular growth rotation in low and higth MP-SN angle faces: a longitudinal study. Angle Orthod., Appleton, v. 67, no. 2, p. 103-110, 1997.

19. MATHEWS, J. R.; WARE, W. H. Longitudinal mandibular growth in children with tantalum implants. Am J Orthod., St. Louis, v. 74, no. 6, p. 633-655, Dec. 1978.

20. NANDA, R. S. Contributions of craniofacial growth to clinical orthodontics. Am. J. Orthod. Dentofacial Orthop., St. Louis, v. 117, no. 5, p. 553-555, May 2000.

21. PERERA, P. S. G. Rotational growth and incisor compensation. Angle Orthod., Appleton, v. 57, no. 1, p. 39-49, Jan. 1987

22. RIEDEL, R. A. The relation of maxillary structures to cranium in maloccusion and in normal occlusion. Angle Orthod., Appleton, v. 22 , no. 3, p. 142-145, 1952

23. RIEDEL, R. A. An analisys od dentofacial relationships. Am. J. Orthod., St. Louis, v. 43, no. 32, p. 103-119, Feb. 1957.

24. SCHUDY, G. F. Post treatment craniofacial growth: Its implications in orthodontics treatment. Am. J. Orthod. Dentofacial Orthop., St. Louis, v. 65, no. 1, p. 38-56, Jan 1974. 
25. SIQUEIRA, V. C. V. et al. Avaliação da recidiva da sobressaliência, em pacientes com Classe, $1^{\text {a }}$ divisão, submetidos a extrações dos quatro primeiros pré-molares: estudo cefalométrico longitudinal, realizado ao início, final e 5 anos após o tratamento ortodôntico. $1^{\text {a }}$ parte: a influência do crescimento craniofacial. Ortodontia, São Paulo, v. 32, n. 2, p. 46-65, maio/ ago. 1999.

26. SIQUEIRA, V. C. V. Avaliação da recidiva da sobressaliência, em pacientes com classe, $1^{\text {a }}$ divisão, submetidos a extrações dos quatro primeiros pré-molares: estudo cefalométrico longitudinal, realizado ao início, final e 5 anos após o tratamento ortodôntico. $2^{a}$ parte: a influência da terapia ortodôntica. Ortodontia, São Paulo, v. 33, n. 1, p. 8-19, jan./abr. 2000.

27. SILVA, M. L. A. Estudo cefalométrico semilongitudinal (fase inicial, final de tratamento e pós-contenção) de jovens brasileiras, leucodermas, tratadas ortodonticamente, portadoras inicialmente de classes I e II, divisão $1^{\text {a }}$ e comparadas com as de oclusão normal. 1986. 100 f. Dissertação (Mestrado)Faculdade de Odontologia de Bauru, Universidade de São Paulo, Bauru, 1986.

28. SOLOW, B. The dentoalveolar compensatory mechanism: background and clinical implications. Br. J. Orthod., Oxford, v. 7, p. $145-161,1980$

29. STEINER, C. C. Cephalometric for you and me. Am. J. Orthod., St. Louis, v. 39, no. 10, p. 729-755, 1953.

30. STEINER, C. C. Cephalometric in clinical practice. Angle Orthod., Appleton, v. 29, no. 1, p. 8-29, Jan. 1959.
31. TWEED, C. H. Indications for extraction of teeth in orthodontic procedure. Am. J. Orthod. Oral Surg., St. Louis, v. 30, no. 8, p. 405-428, Aug. 1944

32. TWEED, C. H. The Frankfourt mandibular plane Angle in orthodontic diagnosis, classification, treatment, planning and prognosis. Am. J. Orthod. Oral Surg., St. Louis, v. 32, no. 4, p. 175-230, Apr. 1946.

33. TWEED, C. H. Evolutionary trends in Orthodontics, past, present and future. Am. J. Orthod. St. Louis, v. 39, no. 2, p. 81-108, Feb. 1953.

34. TWEED, C. H. The Frankfurt mandibular incisor angle (FMIA) in orthodontic diagnosis, treatment, planning and prognosis. Angle Orthod., Appleton, v. 24, no. 3, p.121-169, July 1954

35. TSUNORI, M.; MASHITA, M.; KASAI, K. Relationship between facial types and tooth and bone characteristics of the mandible obtained by CT scanning. Angle Orthod., Appleton, v. 68, no. 6, p. 557-562, 1998.

36. VALADARES NETTO, J.; DOMINGUES, M. H. M. S.; CAPELOZZA FILHO, L. Pesquisa em Ortodontia: bases para a produção e a análise. Rev. Dental Press Ortodon. Ortop. Facial, Maringá, v. 5, n. 4, p. 89-105, jul./ago. 2000

37. WYLIE, W. L. The assesment of anteroposterior dysplasia. Angle Orthod., Appleton, v. 17, n. 3/4, p. 97-109, July/Oct. 1947.

38. WEHRBEIN, H.; BAUER, W.; DIEDRICH, P. Mandibular incisors, alveolar bone, and symphysis after orthodontic treatment. A retrospective study. Am. J. Orthod. Dentofacial Orthop. St. Louis, v. 110, no. 3, p. 239-246, Sept. 1996.
Endereço de correspondência

Vania C. V. Siqueira

Rua José Corder 87 - Jardim Modelo

CEP: 13.400-010 - Piracicaba / SP

E-mail: siqueira@fop.unicamp.br 